\title{
Fstl1 is involved in the regulation of radial glial scaffold development
}

\author{
Rui Liu', Yang Yang ${ }^{1}$, Junhui Shen ${ }^{1}$, He Chen ${ }^{1}$, Qianqian Zhang ${ }^{1}$, Ru Ba' ${ }^{1}$ Yongjie Wei ${ }^{1}$, Kai-Cheng Li²,
} Xu Zhang ${ }^{2^{*}}$ and Chunjie Zhao, ${ }^{1,3^{*}}$

\begin{abstract}
Background: Radial glial cells (RGCs), the instructive scaffolds for neuronal migration, are well characterized by their unique morphology and polarization; these cells extend elongated basal processes to the pial basement membrane (BM) and parallel to one another. However, little is known about the mechanisms that underlie the developmental regulation and maintenance of this unique morphology.

Results: Here, by crossing Fst/1//fl mice with an Ella-Cre line, we identified a new role for the secreted glycoprotein Follistatin like-1 (FSTL1). The ablation of Fst/1 in both of its cortical expression domains, the ventricular zone (VZ) and the pia mater, resulted in RGC morphologic disruption; basal processes were not parallel to each other, and endfeet exhibited greater density and branching. However, Fst/1 deletion in only the VZ in the Emx $1^{\mid R E S c r e} ;$ Fst/ fl/fl line did not affect RGC morphology, indicating that FSTL1 derived from the pia mater might be more important for RGC morphology. In addition, upper-layer projection neurons, not deeper-layer projection neurons, failed to reach their appropriate positions. We also found that BMP, AKT/PKB, Cdc42, GSK3 $\beta$, integrin and reelin signals, which have previously been reported to regulate RGC development, were unchanged, indicating that Fst/1 may function through a unique mechanism.
\end{abstract}

Conclusions: In the present study, we identified a new role for FSTL1 in the development of radial glial scaffolds and the neuronal migration of upper-layer projection neurons. Our findings will improve understanding of the regulation of RGC development and neuronal migration.

\section{Background}

Radial glial cells (RGCs), which serve as progenitor cells and as the scaffolds for neuronal migration, are important for neocortical morphogenesis [1,2]. During cortical development, the soma of an RGC lies in the ventricular zone (VZ), with a specialized apical domain in contact with the ventricular surface, while an elongated basal process extends from its cell body through the entire cortical wall. The tips of its basal processes, which are called the basal endfeet, attach to the pial basement membrane (BM) [1,3]. Despite the diverse functions of RGCs, the mechanisms underlying their development

\footnotetext{
*Correspondence: xu.zhang@ion.ac.cn; zhaocj@seu.edu.cn

${ }^{2}$ Institute of Neuroscience and State Key Laboratory of Neuroscience,

Shanghai Institutes for Biological Sciences, Chinese Academy of Sciences,

Shanghai 200031, China

'Key Laboratory of Developmental Genes and Human Diseases, MOE, Department of Anatomy and Neuroscience, Medical School, Southeast University, Nanjing 210009, China

Full list of author information is available at the end of the article
}

remain unclear. In the $\mathrm{VZ}$, apical signalling molecules, such as beta-catenin, ZO-1, and Cdc42, are reportedly crucial for the integrity of intercellular adhesion, the specialization of the apical membrane domain and the division pattern of RGCs $[4,5]$. The development of polarity and the correct orientation of RGCs' basal processes depend on the activity of adenomatous polyposis coli (APC) and GSK3 [5-7]. Previous studies have provided evidence that the basal radial processes of RGCs receive signals from the meninges and adjacent Cajal-Retzius (CR) cells $[8,9]$. However, the mechanisms that regulate the development of RGC processes remain unclear.

FSTL1 has been reported to be involved in the fate determination and maturation of epithelial cells [10, 11]. However, the function of Fstl1 during cortical development remains unknown. Previously, we have observed that Fstl1 is enriched in the VZ and the pia mater of the developing cerebral cortex [12]. Considering the unique morphology of RGCs and the distinct expression pattern

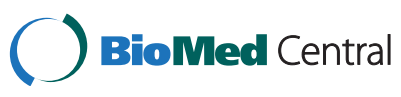

(c) 2015 Liu et al. Open Access This article is distributed under the terms of the Creative Commons Attribution 4.0 International License (http://creativecommons.org/licenses/by/4.0/), which permits unrestricted use, distribution, and reproduction in any medium, provided you give appropriate credit to the original author(s) and the source, provide a link to the Creative Commons license, and indicate if changes were made. The Creative Commons Public Domain Dedication waiver (http://creativecommons.org/publicdomain/zero/1.0/) applies to the data made available in this article, unless otherwise stated. 
of FSTL1, it is quite plausible that Fstl1 is involved in the development of RGCs. In the present study, we report that ablation of Fstl1 in both the VZ and the pia mater led to abnormal radial scaffold morphology and further led to the abnormal distribution of cortical pyramidal neurons.

\section{Results}

\section{Disruption of Fstl1 results in an abnormal distribution of} cortical upper-layer neurons

To better understand the development of radial glial scaffolds and to address the function of Fstll during cortical morphogenesis, Fstl1 was ablated by crossing the EIIa-Cre line with Fstll fllfl. In situ hybridization showed a dramatic reduction of Fstl1 mRNA in the VZ and the pia mater in $F_{s t l 1^{-1-}}$ brains at E16.5 (Fig. 1a-b'). The disruption efficiency was also confirmed by quantitative real-time polymerase chain reaction (qRT-PCR) (Fig. 1c). Because the homozygous null pups died within a few hours of birth, the observations were conducted at embryonic stages. HE staining at E18.5 showed a slight reduction in the thickness of the cortical wall in the $F_{s t l 1^{-/}}$embryos compared with that of the WT cortical walls, but the overall architecture of the brain did not appear to be severely affected (Fig. 1d-f'). The cortical neurons, especially the upper-layer neurons, were not tightly arranged, but the overall lamination did not appear to be obviously disturbed (Fig. 1e-f'). These findings suggest that the loss of Fstl1 affects the accurate localization of cortical neurons.
To assess which groups of neurons were affected during cortical development, the layer-specific markers Brn2 (II-III), Cux1 (layers II-IV), Ctip2 (V-VI), and Tbr1 (VI) were used. At E18.5, many $\mathrm{Cux}^{+}$and $\mathrm{Brn}^{+}$cells still resided in the intermediate zone (IZ) and subplate in the $F s t l 1^{-/}$mice (Fig. 2a' and b'), but in the WT mice, the majority of the $\mathrm{Cux}^{+}$and $\mathrm{Brn}^{+}$cells had arrived at the upper cortical plate (Fig. 2a and b). Double immunostaining for Cux1 and Ctip2 also showed that at E18.5, a number of $\mathrm{Cux}^{+}$neurons remained in deeper cortical areas in the $\mathrm{Fstl1}^{-/-}$mice compared with the WT mice (Fig. 2c-d'). The statistical analysis showed that at E18.5, only $32 \%$ of the Cux $1^{+}$neurons had arrived in the upper area in the $\mathrm{Fstl1}^{-/-}$cortex compared with $44 \%$ in the WT cortex; in addition, $35 \%$ of the $\mathrm{Cux} 1^{+}$neurons were accumulated in the lower area in the $\mathrm{Fstl1}^{-1-}$ cortex but only $24 \%$ were in the WT cortex (Fig. 2e). However, the total number of $\mathrm{Cux}^{+}$neurons did not differ significantly between the Fstl1 ${ }^{-/-}$and WT mice (Fig. 2f). Our data showed that the migration of upper-layer projection neurons was disrupted after Fstl1 deletion. In the deeper-layer projection neurons, the distributions of $\mathrm{Tbr}^{+}$layer VI neurons and Ctip2-high expression layer Va neurons in the $F_{s t l 1^{-1}}$ mice were comparable with those in the WT mice (Fig. 2g-i'), and the total numbers of $\mathrm{Tbr}^{+}$neurons and Ctip2 high-expression neurons did not differ (Fig. $2 j$ and $k$ ). The lamination of the deeper layers seemed similar between the $F s t l 1^{-/-}$and WT mice, and there was a clear boundary between layer VI and layer Va (Fig. 2g-i').

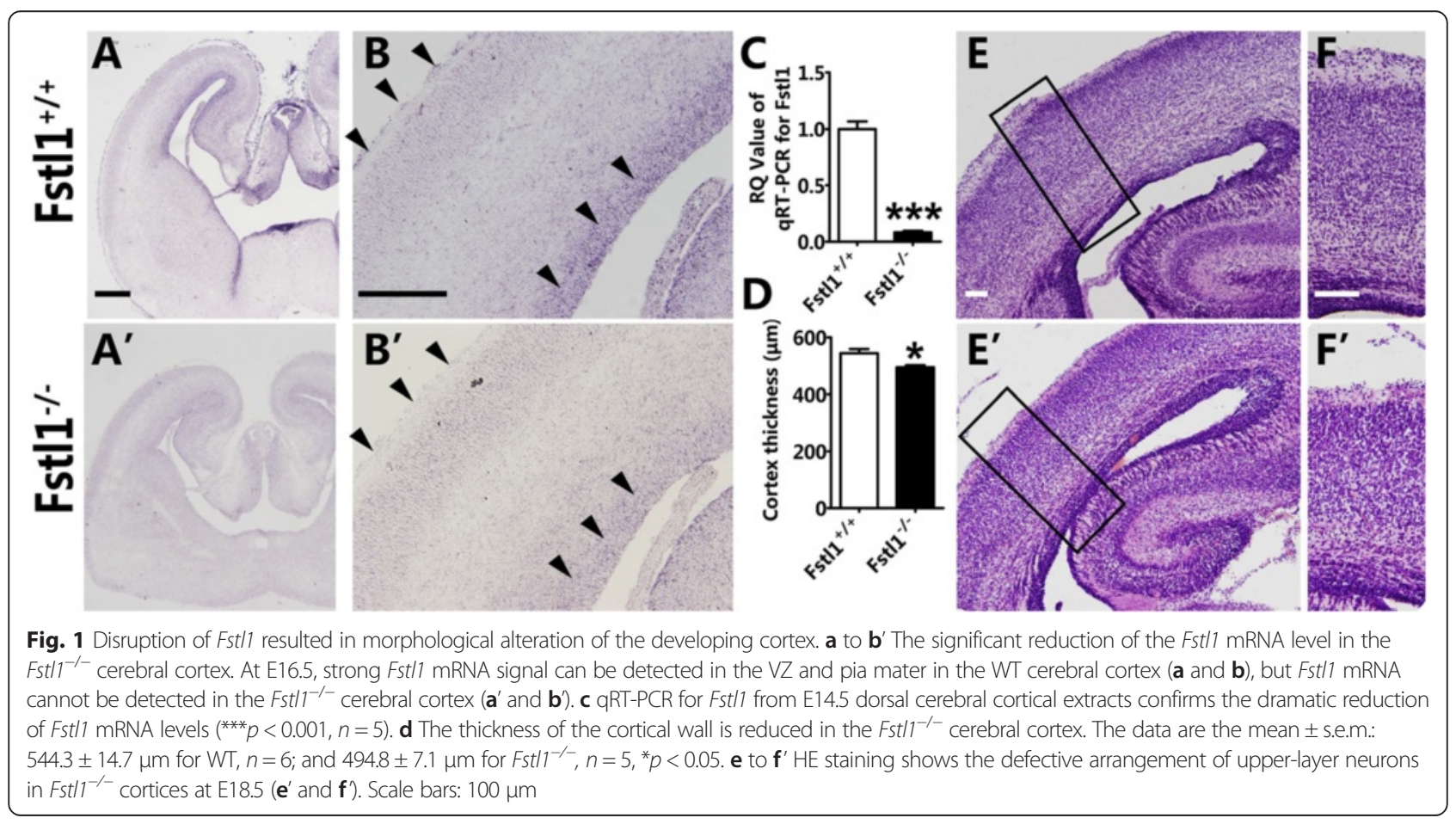




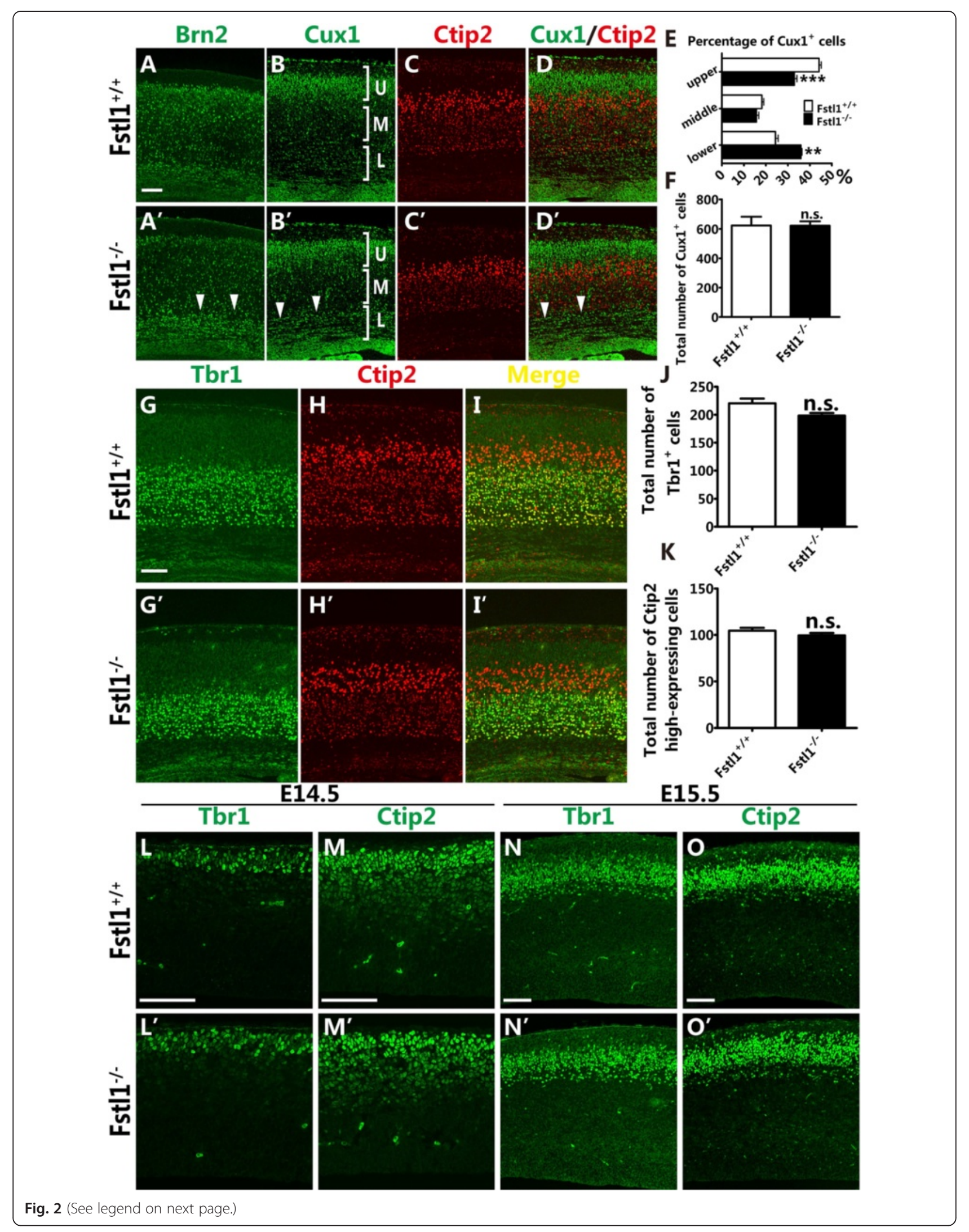




\section{(See figure on previous page.)}

Fig. 2 Disruption of Fst/7 results in an abnormal distribution of upper-layer but not deeper-layer cortical neurons. a to d' The abnormal distribution of upper-layer neurons in the Fst/1 ${ }^{-1-}$ cerebral cortex. Immunostaining for Brn2 (arrowheads, [a] ) and Cux1 (arrowhead, [b']) at E18.5 shows many upper-layer neurons still residing in the IZ and subplate after Fst/1 ablation (arrowhead in $\mathbf{a}^{\prime}$ and $\mathbf{b}^{\prime}$ ). Co-immunostaining for Cux1 (b, $\mathbf{b}^{\prime}, \mathbf{d}$ and $\mathbf{d}$ ) and Ctip2 (c, $\mathbf{c}^{\prime}, \mathbf{d}$ and $\mathbf{d}^{\prime}$ ) at E18.5 shows the abnormal distribution of upper-layer neurons in the deeper area. e The distribution of Cux $1^{+}$neurons at E18.5. More Cux ${ }^{+}$ neurons accumulated in the lower area at E18.5 (24.31 $\pm 1.26 \%$ for WT, $n=4 ; 35.94 \pm 0.48 \%$ for Fst $\left.11^{-1}, n=4, * * 0<0.01\right)$, while fewer Cux $1^{+}$neurons were present in the upper area (44.14 $\pm 1.03 \%$ for WT, $n=4 ; 32.98 \pm 1.16 \%$ for $\left.F s t 11^{-1-}, n=4,{ }^{* * *} p<0.001\right)$. $\mathbf{f}$ The total number of Cux ${ }^{+}$neurons per area at E18.5. The data are the mean \pm s.e.m.: $623.5 \pm 58.5$ for WT, $n=4$; and $622.3 \pm 29.1$ for Fst $11^{-1-}, n=4, p=0.99$. $\mathbf{g}$ to $\mathbf{i}^{\prime}$ Double immunostaining for Tbr1 and Ctip2 at E18.5 showed that the distributions of layer VI and layer Va neurons are unchanged in the Fst $11^{-1-}$ cortex compared to the WT cortex. $\mathbf{j}$ and $\mathbf{k}$ The total numbers of Tbr1 ${ }^{+}$layer VI neurons (j) and layer Va neurons with high Ctip2 expression per area at E18.5 (k). The Tbr $1^{+}$neuron data are presented as the mean \pm s.e.m.: $220.5 \pm 8.4$ for $W T, n=4$; and $198.4 \pm 4.7$ for Fst $/ 1^{-1}, n=4, p=0.07$. The high Ctip2 expression neuron data are presented as the mean \pm s.e.m.: $104.5 \pm 3.0$ for WT, $n=5$; and $99.4 \pm 2.6$ for Fst/1 ${ }^{-/}, n=5, p=0.24$. I to $\mathbf{o}^{\prime}$ The comparable distribution of deeper-layer neurons at early embryonic stages in WT and $F_{s t 1} 11^{-/-}$mice. Immunostaining for the deeper-layer neuronal markers Tbr1 (I, I', $\mathbf{n}$ and $\left.\mathbf{n}^{\prime}\right)$ and Ctip2 (m, $\mathbf{~ m}^{\prime}, \mathbf{O}$ and $\left.\mathbf{o}^{\prime}\right)$ at E14.5 (I to $\mathbf{~ m}^{\prime}$ ) and E15.5 (n to o') shows that the migration and distribution of deeper-layer neurons are unchanged in the $\mathrm{Fst}_{1} \mathrm{~T}^{-/-}$cortex compared to the WT cortex. Scale bars: $100 \mu \mathrm{m}$

To examine whether the radial migration of deeperlayer neurons was delayed at earlier embryonic stage and finally recovered at E18.5, cortices were harvested at E14.5 and E15.5 and immunostained with the deeperlayer-specific markers Tbr1 and Ctip2. Our data revealed that $\mathrm{Tbr}^{+}$and $\mathrm{Ctip}^{+}$neurons in the $\mathrm{Fstl1}^{-1-}$ cortex were positioned appropriately in the emerging cortical plate at E14.5 and in the deeper part of the cortical plate at E15.5, comparable with the positions observed in the WT cortex, as shown in Fig. 2l-o'. Thus, the migration of deeper-layer projection neurons was not obviously disrupted. Taken together, our data showed that the migration of upper-layer projection neurons was more severely impaired at perinatal stages following Fstll ablation.

To test whether the ablation of Fstl1 resulted in cortical interneuron defects, we examined the distribution of interneurons derived from the medial ganglionic eminence (MGE) and caudal ganglionic eminence (CGE). In situ hybridization for the neuropeptide somatostatin (SST) and the LIM homeobox protein 6 (Lhx6) showed that the distribution of MGE-derived interneurons was unchanged in $\mathrm{Fstl}^{-/-}$mice compared to WT mice. Immunostaining for COUP-TFII also indicated that the overall migration and lamination of CGE-derived interneurons were similar to those of the WT interneurons at E18.5 (data not shown). Our data demonstrated that disruption of Fstl1 had no obvious effects on cortical interneuron development.

To further elucidate the role of Fstl1 in neuronal migration, 5-bromo-2-deoxyuridine (BrdU) birth-dating was employed. BrdU was injected at E12.5 to label the deeperlayer neurons or at E14.5 to label the upper-layer neurons. The brains were then harvested at E18.5. In the mutants, the distribution of deeper-layer $\mathrm{BrdU}^{+}$neurons born at E12.5 was similar to that in the WTs (Fig. 3a and a'). In the WT mice, $46.3 \pm 2.3 \%$ of the $\mathrm{BrdU}^{+}$cells that were labelled at E14.5 populated the upper part of the cortex; however, this percentage was reduced to $35.3 \pm 3.0 \%$ in the $\mathrm{Fstl1}^{-/-}$mice. The $\mathrm{BrdU}^{+}$cells were distributed more evenly throughout the cortex, and a population of $\mathrm{BrdU}^{+}$ cells remained in the deeper cortical layers and IZ in the $F s t l 1^{-/}$mice. In the WT mice, approximately $25.5 \pm$ $1.6 \%$ of the $\mathrm{BrdU}^{+}$cells were distributed in the deeper layers, but this percentage increased to $33.3 \pm$ $1.3 \%$ in the Fstl1 ${ }^{-/-}$mice (Fig. $3 \mathrm{~b}$ and b'). To further assess the migration of deeper-layer neurons at earlier developmental stages, BrdU was injected at E12.5, and the brains were then harvested at E14.5. In the mutants, the distribution of $\mathrm{BrdU}^{+}$neurons born at E12.5 was similar to that in the WTs (Fig. 3c and c'). These results indicate that Fstll dysfunction resulted in mis-positioning of the upper- but not deeper-layer projection neurons.

To examine whether Fstl1 affects neuronal proliferation, acute BrdU labelling was performed at E14.5 and E16.5 (Fig. 3d-e'). The numbers of BrdU-labelled cells did not differ between the $\mathrm{Fstl1}^{-1-}$ pups and their WT littermates (E14.5, $172.4 \pm 5.1$ for WT, $n=5$ vs $173.5 \pm$ 7.2 for $F_{s t l 1}{ }^{-/}, n=5$; E16.5, $69.5 \pm 2.4$ for WT, $n=3$ vs $73.5 \pm 2.1$ for $F s t l 1^{-1-}, n=3$ ) (Fig. 3f and g), and this result was further confirmed by immunostaining for the pan-proliferation marker Ki67 and the mitotic phase marker phospho-histone H3 (pHH3) (Fig. 3h-i'), which suggests that the deletion of Fstl1 does not affect the proliferation of progenitor cells. Because upper-layer cortical neurons are primarily derived from intermediate progenitor cells (IPCs) rather than directly from RGCs, we next examined the number of IPCs by immunostaining for Tbr2 [13] and found no obvious differences (Additional file 1: Figure S1), which indicates that the neurogenesis of the upper-layer neurons was also not affected. Additionally, immunostaining for activated Caspase 3 was performed at E14.5, and an obvious excess of cell death was not detected (data not shown).

\section{The development of radial glial scaffolds is disrupted in Fst $11^{-1-}$ cerebral cortices}

Because the upper-layer neurons reach their final destinations by migrating along radial glial scaffolds [14], we 

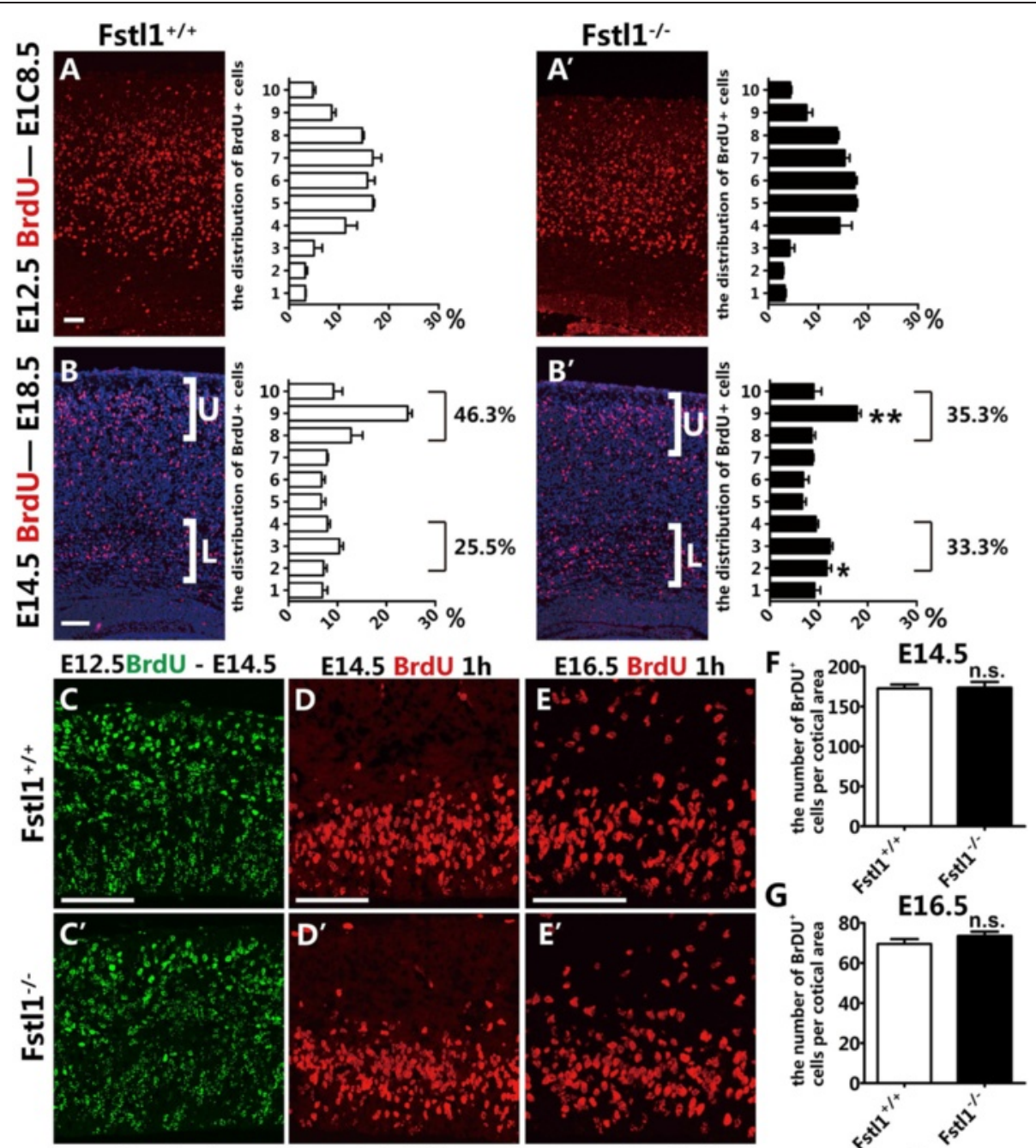

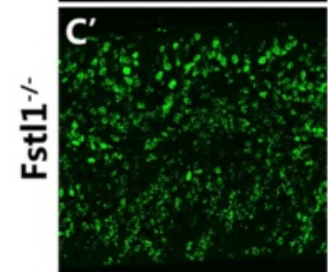

Fstl1+/+

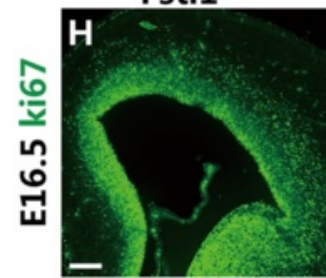

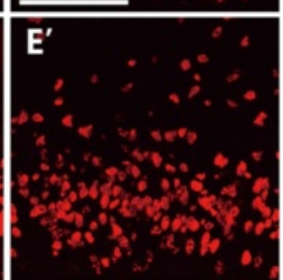

Fstl1 1++
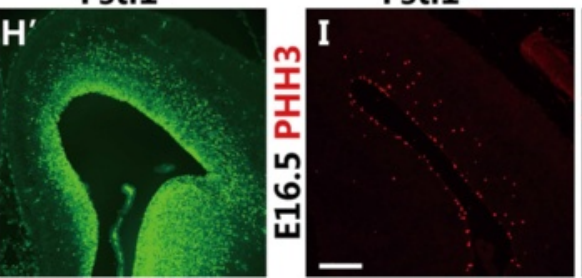

Fstl1\%

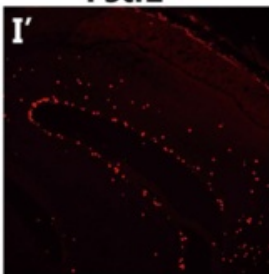

Fig. 3 Loss of Fst/17 results in aberrant positioning of upper-layer cortical neurons. a to $\mathbf{b}^{\prime}$ Although similar distributions of E12.5-born cells are observed

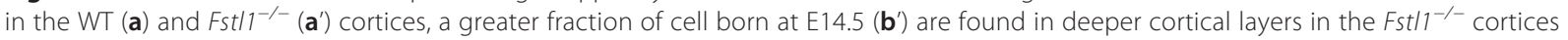
compared to the WT cortices (b). The percentages of cells in the upper layers (bins 8-10) and IZ (bins 2-4) are indicated in the histograms. $n=3$ per genotype per experimental condition. ${ }^{*} p<0.05,{ }^{* *} p<0.01$. The error bars represent the s.e.m. $\mathbf{c}$ and $\mathbf{c}^{\prime}$ The migration of early-born deeper-layer neurons is unchanged in the Fst $/ 1^{-/-}$cortex (c) compared to that in the WT cortex (d). BrdU was injected at E12.5, and the brains were then harvested at E14.5. $\mathbf{d}$ to $\mathbf{e}^{\prime}$ The number of BrdU ${ }^{+}$cells was similar in the WT (d and e) and Fst $/ 1^{-/}$cortices (d' and e') at E14.5 (d and d') and E16.5 (e and $\mathbf{e}^{\prime}$ ). $\mathbf{f}$ and $\mathbf{g}$ The number of BrdU ${ }^{+}$progenitor cells per entire cortical section at E14.5 (f) and E16.5 (g). The data are the mean \pm s.e.m. At E14.5, $172.4 \pm 5.1$ for WT, $n=5$; and $173.5 \pm 7.2$ for Fst $/ 1^{-1-}, n=5, p=0.91$. At E16.5, $69.5 \pm 2.4$ for WT, $n=3$; and $73.5 \pm 2.1$ for Fst $/ 1^{-1-}, n=3, p=0.28$. h to i' Immunolabelling with anti-Ki67 (h and $\mathbf{h}^{\prime}$ ) and anti-pHH3 (i and $\mathbf{i}^{\prime}$ ) antibodies confirmed that the proliferation of progenitor cells was not obviously changed at E16.5. Scale bars: $100 \mu \mathrm{m}$

next investigated whether the positional defect of the upper-layer neurons in the $F s t l 1^{-1-}$ mice was due to an abnormality in the RGCs. Immunostaining for nestin and BLBP, which are standard markers for RGCs, was performed. At E12.5, the nestin-stained radial glial scaffolds in the $F s t l 1^{-/-}$mice appeared to be similar to those in the WT mice, with many radially distributed processes extending throughout the cortical wall (Fig. 4a and a'). 

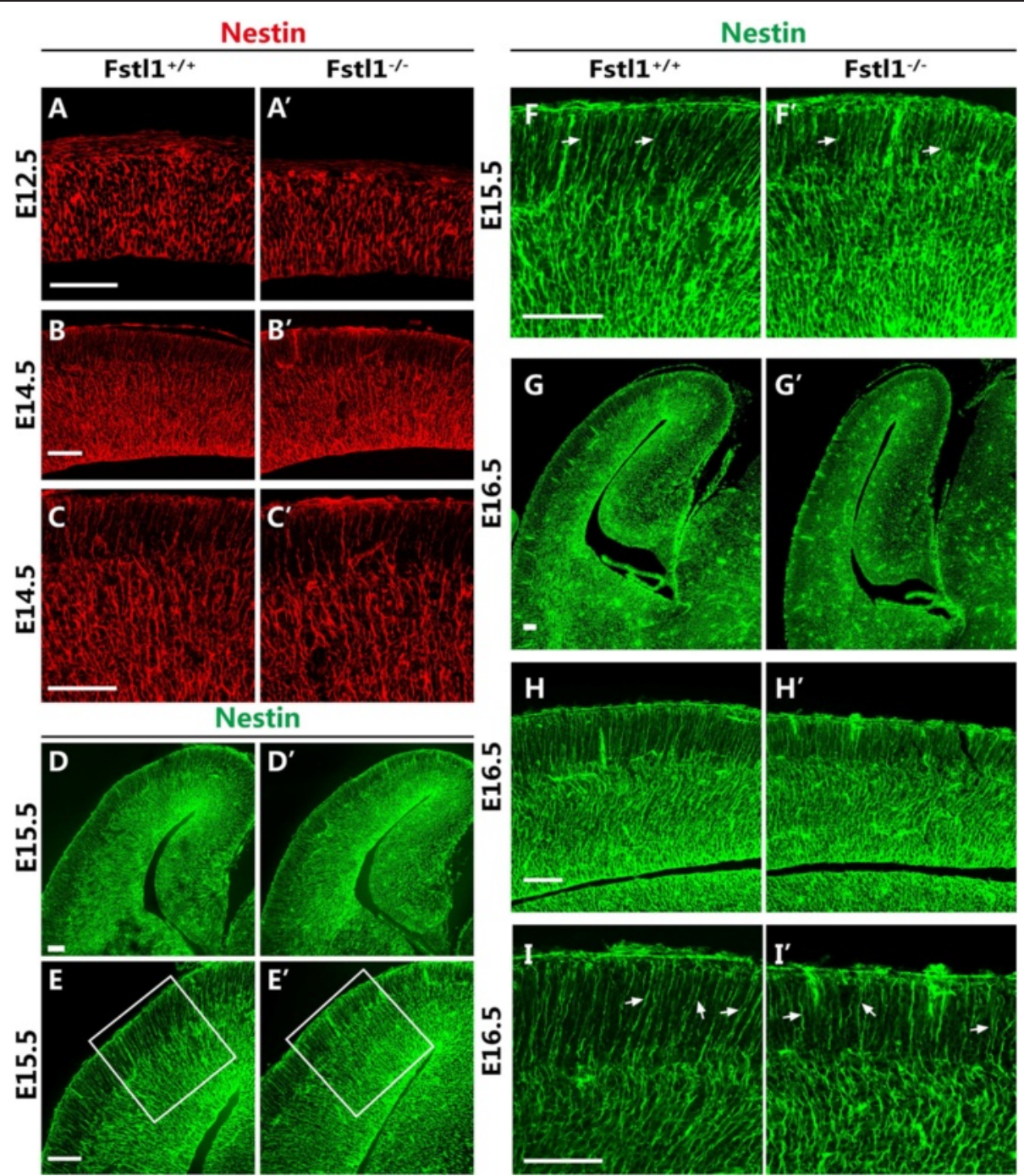

Fig. 4 Fst/1 deletion impairs the morphology of radial glial processes. a to i' Nestin immunostaining at E12.5 (a and $\left.\mathbf{a}^{\prime}\right)$, E14.5 (b to $\mathbf{c}^{\prime}$ ), E15.5 (d to $\left.\mathbf{f}^{\prime}\right)$ and E16.5 (g to i'). At E12.5, the development of radial glial fibres was comparable between the WT (a) and Fst/1 ${ }^{-1-}$ (a') cortices. b to $\mathbf{c}^{\prime}$ At E14.5, many long, parallelly distributed processes spanning the entire cerebral cortex were observed in the WT brains (b and $\mathbf{c})$. In the Fst/1 $1^{-1-}$ brains, many radial processes were not parallel to one another ( $\mathbf{b}^{\prime}$ and $\mathbf{c}^{\prime}$ ). $\mathbf{d}$ to i' At E15.5 and E16.5, the same phenomenon was detected in the RGCs. Scale bars: $100 \mu \mathrm{m}$

However, although many long, radially distributed processes spanned the entire cerebral cortex in the WT mice at E14.5, numerous short radial processes failed to extend perpendicularly through the entire cerebral cortex towards the pial BM in the Fstll ${ }^{-/-}$mice, and the radial scaffolds were not fasciculated as tightly as in the WT mice (Fig. 4b-c'). Similar defects in the radial glial scaffolds were detected at E15.5 and E16.5 (Fig. 4d-i'). Immunostaining for BLBP further confirmed the defects at E15.5 and E16.5 (Fig. 5a-d'). At E18.5, the defects in the RGC processes were still apparent (Fig. 5e and e'). To examine whether the radial glial progenitor pool was affected, immunostaining for the transcription factor Pax6, which labels RGCs $[13,15]$, was performed, and the progenitor pool was found to be relatively normal (Fig. $5 f$ and $f^{\prime}$ ).
To further study the dysmorphic radial glia in the absence of Fstl1, the radial glial scaffolds were labelled with DiI at E15.5 and observed with a standard fluorescence microscope (Fig. 6a and a') or a confocal microscope (Fig. 6b-d'). In the WT brains, the RGC processes were oriented parallel to one another and orthogonal to the pial BM (Fig. 6b and c), and their basal endfeet were less branched (Fig. 6a-d). However, in the $F s t l 1^{-1-}$ cortices, many RGC processes were not parallel to one another (Fig. 6a'-d'). Furthermore, the branches of the basal endfeet were more intricate and were located farther away from the pial surface than those in the WT cortices (Fig. 6c-d'). Thus, the disruption of Fstl1 results in abnormal RGC development. 


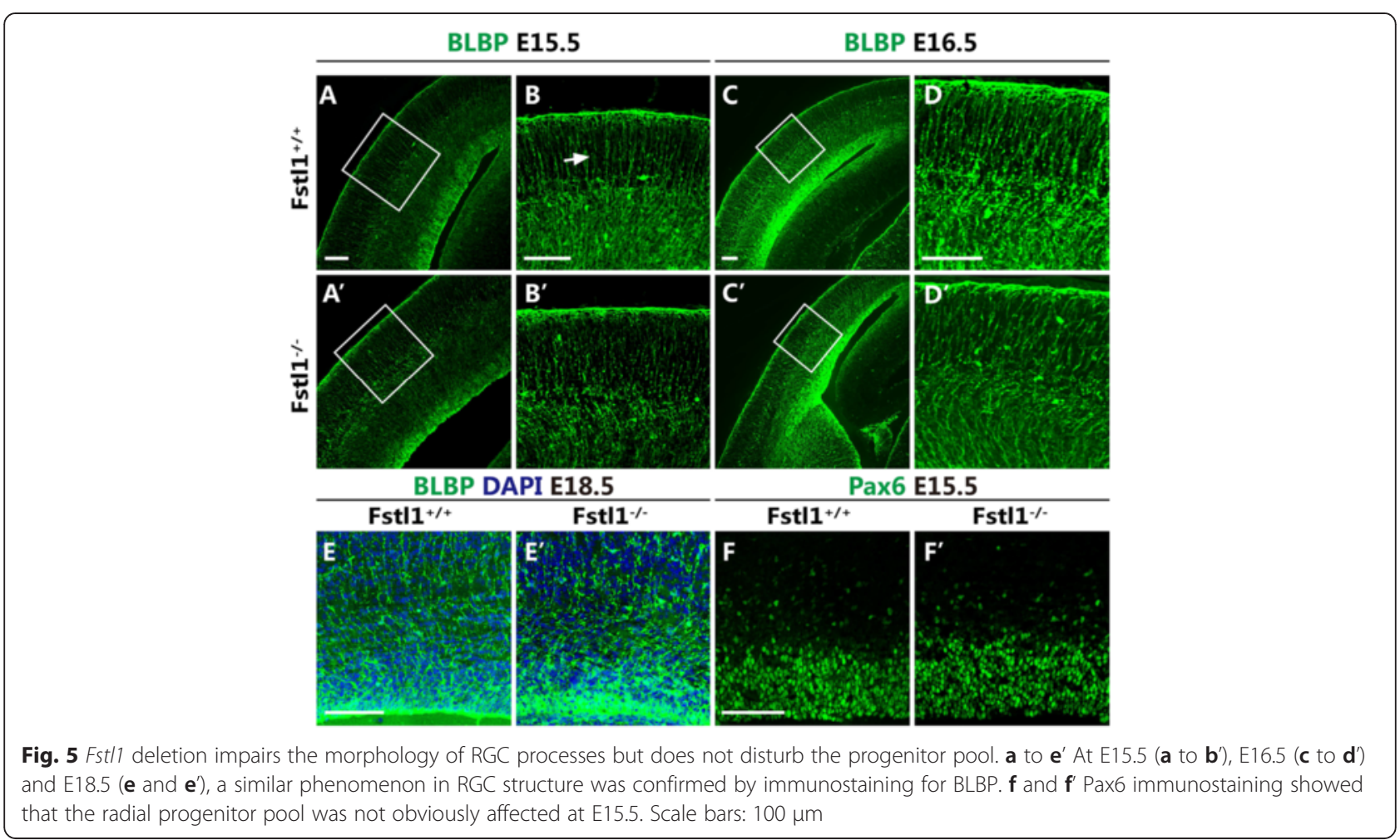

\section{Basal rather than apical polarity of RGCs is altered in the} absence of Fst/1

A defining feature of RGCs is their apical-basal polarity. Recent studies have indicated that disruption of the polarity of RGCs disturbs both the morphology of radial glial processes and neuronal migration [5]. The tumour suppressor APC is highly concentrated at the tips of the processes and in the somas of RGCs and is required for the maintenance and extension of the radial glial processes [7]. To test whether the polarity of RGCs was altered after Fstl1 ablation, immunostaining for APC was conducted. At E12.5, APC was highly expressed at the pially directed tips of the RGCs and on the apical surface of the VZ in both the Fstl1 ${ }^{-/-}$and WT mice (Fig. 7a and a'). However, at E14.5, a broader APC-enriched band was observed in the Fstl1 ${ }^{-/-}$subpial region compared to that in the WT region (Fig. $7 \mathrm{~b}-\mathrm{d}$ ), which is consistent with the radial glial endfeet defect observed through DiI labelling (Fig. 6a-d'). Upon examination of the integrity of the pial BM, the attachment site for the RGCs' basal endfeet [16], no abnormalities were detected in the Fstl1 $^{-/-}$mice, as demonstrated by laminin A immunoreactivity (Additional file 2: Figure S2 and data not shown). These findings demonstrated that the endfeet of the pially directed, polarised radial glial processes could not be established correctly in the absence of Fstl1, although the pial BM itself was not affected.

Previous studies have shown that $\beta$-catenin, which is localized at the apical adherens junctions, modulates appropriate expansion of the radial progenitor population [4, 17]. ZO-1, which is a tight junction-associated protein, is enriched at the lateral membrane domain of the interphase radial glial apical domain [18]. To examine whether the apical polarity of the RGCs was affected, immunostaining for $\beta$-catenin and ZO-1 was performed. Relatively normal $\beta$-catenin and ZO-1 expression patterns were observed in the Fstl1 ${ }^{-/-}$brains (Fig. 7e-f'), which suggests that the apical polarity was not grossly altered. Taken together, the results showed that the ablation of Fstl1 led to disruption of the basal rather than the apical polarity of RGCs.

\section{Ablation of Fst/1 in both the VZ and the pia mater rather} than in only the VZ leads to abnormal RGC morphology

Because Fstl1 mRNA is found in both the pia mater and the VZ [12], we sought to determine whether FSTL1 derived from the $\mathrm{VZ}$ or the pia mater is more important for the maintenance of polarity in RGCs. Emx $1^{\text {IREScre mice }}$ [19] were employed to disrupt Fstl1 only in the VZ and not in the pia mater (Fig. 8a-b'). Unexpectedly, no apparent abnormalities were observed in the cortex of Emx $1^{\text {IRESCre }}$; $F_{s t l} f^{f l f l}$ mice (Fig. 8c and $\mathbf{c}^{\prime}$ ). Both upper-layer and deeperlayer neurons were nicely laminated (Fig. 8d-h'). BrdU birth-dating showed that in the $F s t l 1^{-/-}$mice, the final distribution of upper-layer neurons born at E15 was comparable with that in the WT mice (Fig. $8 \mathrm{~g}$ and g'). At E15.5, the radial glial scaffolds, viewed by immunostaining for BLBP, appeared similar to those of the WT mice, with 


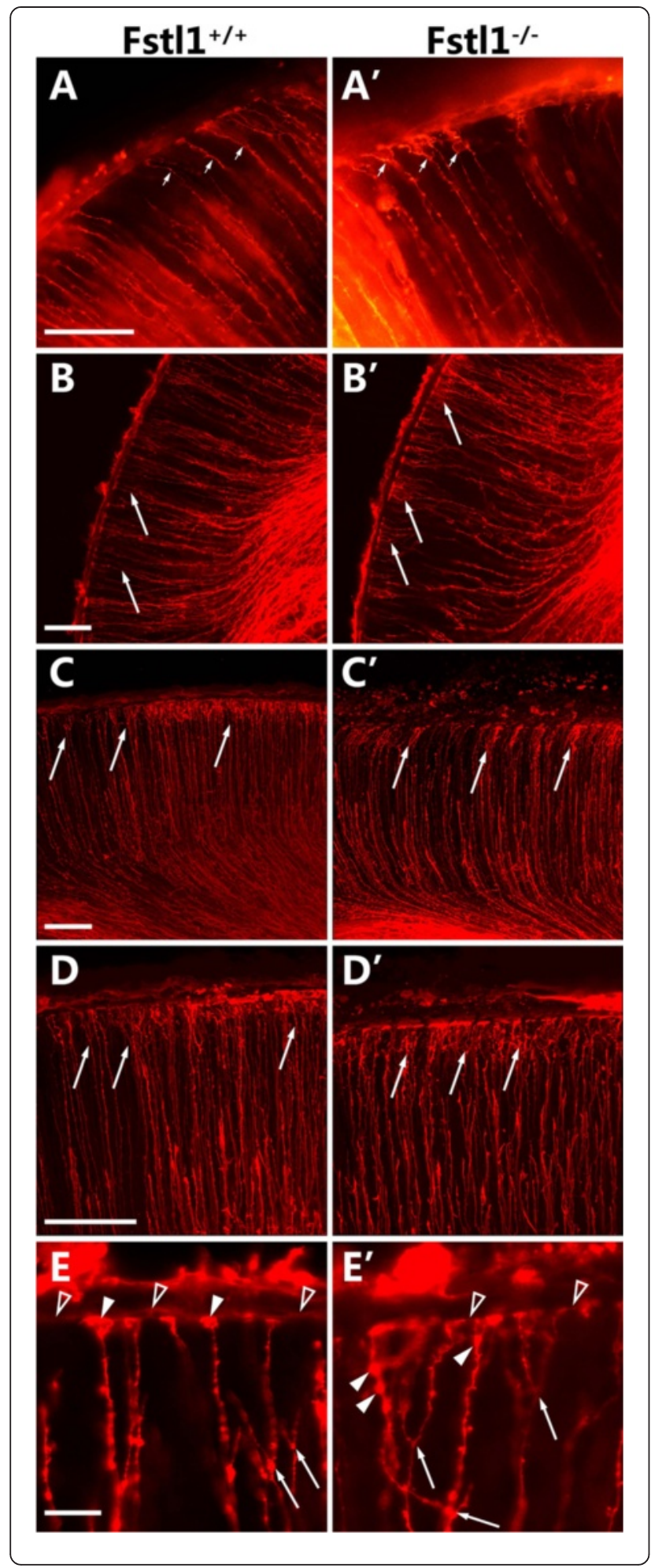

Fig. 6 Dysmorphic RGCs with defective branches near the pial BM in the Fstl1-/- mice. RGC scaffolds are shown by Dil labelling at E15.5. (a and $\mathbf{a}^{\prime}$ ) Images were captured with an Olympus BX61 microscope and a DP71 digital camera. (b and $\mathbf{b}^{\prime}$ ) Images were captured with an FV1000 Olympus confocal microscope. (c to d') The images are a projection of an 80- $\mu \mathrm{m}$ z-stack collected on an Olympus FV1000 confocal microscope. In the WT cortices, the RGC processes were aligned in parallel and were less branched (a and $\mathbf{c}$ ). In the WT cortices, the branches of RGC processes were near the pial BM (d). In the Fstl1-/- cortices, many RGC processes were not parallel to one another $\left(\mathbf{b}^{\prime}-\mathbf{d}^{\prime}\right)$, and the branching of their endfeet was more intricate (arrows in $\mathbf{a}^{\prime}-\mathbf{d}^{\prime}$ ). (e and $\mathbf{e}^{\prime}$ ) The images were high magnification of RGC scaffold endfeet. In the WT cortices, the endfeet of RGC processes (arrowhead in e) attached to the pial BM (open arrowhead in e). In the Fstl1-/- cortices, many RGC scaffold endfeet

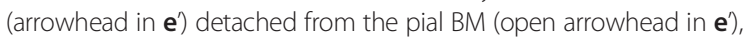
and the branching of RGC processes was more intricate (arrows in $\mathbf{e}^{\prime}$ ). Scale bars: $100 \mu \mathrm{m}$ for $\mathbf{a}-\mathbf{d}^{\prime} ; 20 \mu \mathrm{m}$ for $\mathbf{e}$ and $\mathbf{e}$

many radially distributed processes that spanned the entire cortical wall and were oriented parallel to one another (Fig. 8i and i'). The Pax6 $6^{+}$RGC pool was also unchanged (Fig. $8 \mathrm{j}$ and $\mathrm{j}^{\prime}$ ). Furthermore, neither the distribution nor the intensity of APC staining was affected (Fig. 8k and k'). These results demonstrated that conditional disruption of Fstl1 in the VZ was not sufficient to cause the phenotype observed in the Fstl1 ${ }^{-/-}$brains. Together, our data suggest that FSTL1 derived from the VZ is not necessary for the maintenance of the morphology of RGC processes and that FSTL1 derived from the pia mater is more important.

The possible mechanisms of Fst/1 underlying the development of radial glial scaffolds

BMP signalling may be one of the downstream targets of FSTL1 [20, 21], and the deletion of BMP7 results in less radial glia attachment to the pial BM, reduced cortical thickness and impaired neuronal migration [22, 23]. Therefore, we first examined the activity of the canonical BMP signalling pathway by examining the level of phosphorylated SMAD1/5/8. Western blot analysis indicated that the phosphorylation levels in $\mathrm{Fstl1}^{-/}$mice were similar to those in WT mice (Additional file 3: Figure S3A). BMP7 qRT-PCR further confirmed that the transcription level of BMP was also unchanged (Additional file 3: Figure S3B). The AKT/ PKB signalling pathway is another target of FSTL1. Previous studies have shown that FSTL1 protects cells from apoptosis and induces angiogenesis via phosphorylated AKT [24-26]. However, qRT-PCR and immunoblotting assays showed that the level of AKT was not changed in $F_{s t l 1}{ }^{-1-}$ brains (Additional file 3: Figure S3A and 3C). Furthermore, the activity of the AKT signalling pathway, as monitored by AKT phosphorylation at Ser473, was also unchanged (Additional file 3: Figure S3A). A previous study has shown that Etv5 is a critical target of the FGF/ MAPK signalling pathway and has a role in the 

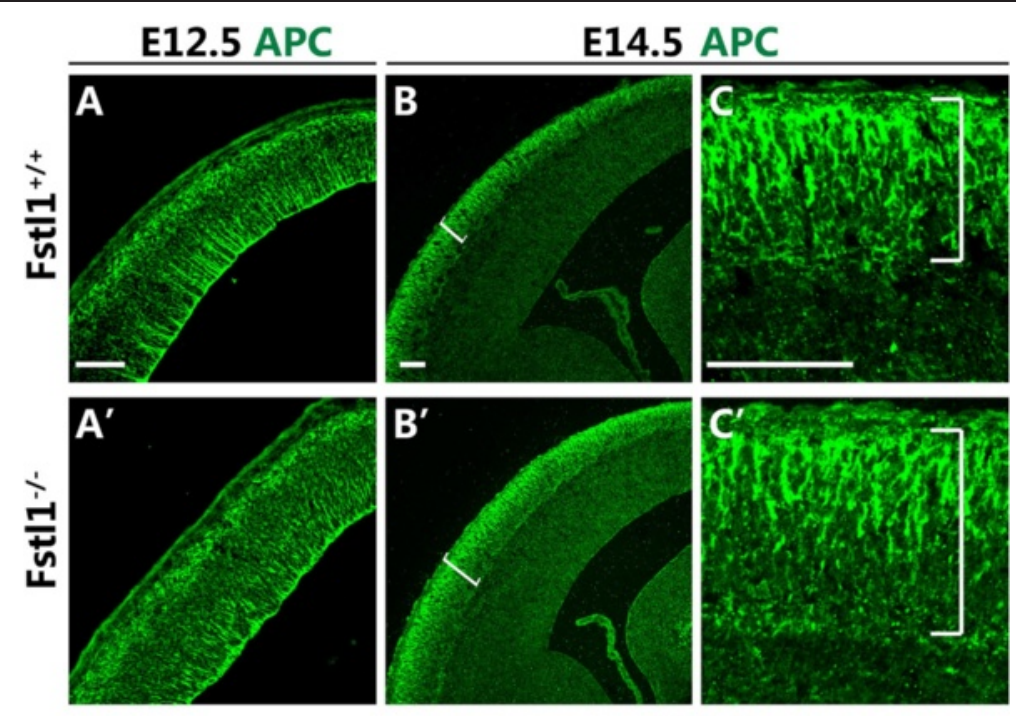

E15.5

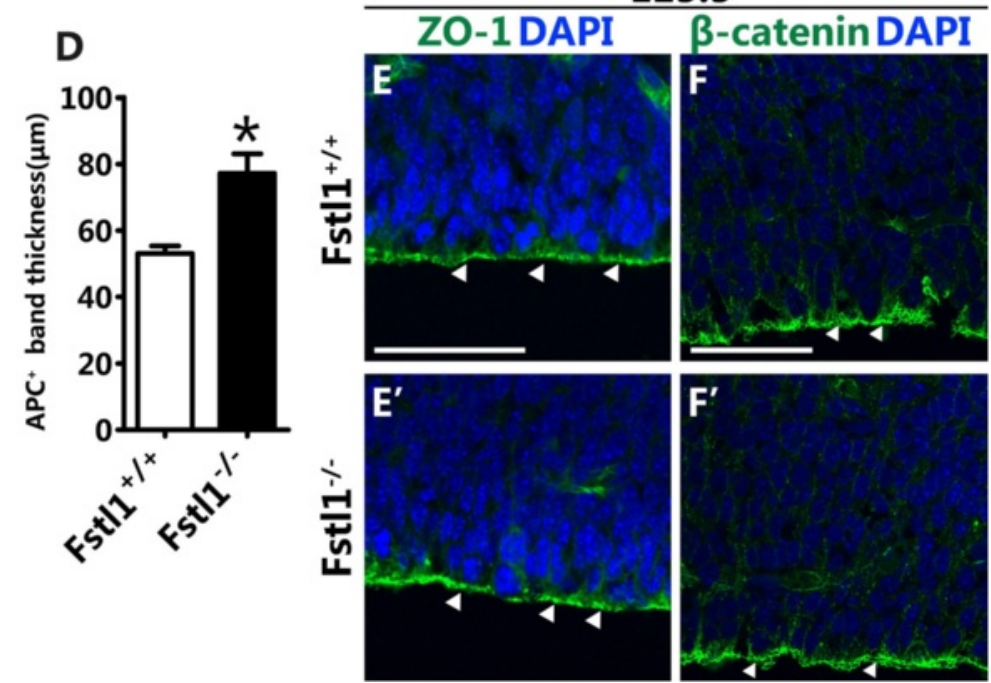

Fig. 7 The basal rather than the apical polarity of RGCs is altered in Fst/1 ${ }^{-1-}$ mice. a and $\mathbf{a}^{\prime}$ At E12.5, APC is highly expressed at the pially directed tips of the RGCs and at the apical BM of the VZ. The expression pattern of APC is comparable between the WT (a) and Fst/1 ${ }^{-1-}$ (a') brains. $\mathbf{b}$ to $\mathbf{c}^{\prime}$ At E14.5, a broader APC-positive band was observed in Fst/1 ${ }^{-1-}$ ( $\mathbf{b}^{\prime}$ and $\mathbf{c}^{\prime}$ ) compared to that of the WT (b and $\mathbf{c}$ ). $\mathbf{c}$ and $\mathbf{c}^{\prime}$ Higher-power views of the boxed areas in $\mathbf{b}$ and $\mathbf{b}^{\prime}$. $\mathbf{d}$ The thickness of the $\mathrm{APC}^{+}$band was reduced in the $F s t / 1^{-1-}$. Data are presented as the mean \pm s.e. $\mathrm{m}$. for the $\mathrm{WT}(53.06 \pm 2.62 \mu \mathrm{m}, n=3$ ) and Fst $11^{-1-}(77.20 \pm 5.87 \mu \mathrm{m}, n=3)$ cortices. ${ }^{*} p<0.05$. e to $\mathbf{g}^{\prime}$ Immunolabelling with anti-ZO-1 (e and $\left.\mathbf{e}^{\prime}\right)$ and anti- $\beta$-catenin (f and $\left.\mathbf{f}^{\prime}\right)$ at E15.5 showed that the apical polarity of the RGCs was not obviously altered. Scale bars: $100 \mu \mathrm{m}$ for a-c', $\mathrm{f}$ and f'; $50 \mu \mathrm{m}$ for $\mathbf{e}$ and $\mathbf{e}^{\prime}$

specification of RGCs [27]. However, neither the expression pattern nor the mRNA level of Etv 5 differed between the WT and $F s t l 1^{-/-}$mice (Additional file 3: Figure S3D, D' and E). Reelin, which is secreted by CR cells, has been reported to be important for the development of radial scaffolds $[28,29]$. However, neither the transcriptional level of reelin nor the distribution of Reelin ${ }^{+} \mathrm{CR}$ cells was altered in $\mathrm{Fstl1}^{-/-}$mice (Additional file 3: Figure S3F to $\left.G^{\prime}\right)$. Additionally, we examined the mRNA levels of Cdc42 and GSK3 $\beta$, which have been reported to modulate distinct aspects of radial glial process organization and function, but no differences were detected between the WT and $\mathrm{Fstl1}^{-/-}$mice (Additional file 3: Figure $\mathrm{S} 3 \mathrm{H}$ and I).
Integrins are critical for the integrity of the pial $\mathrm{BM}$, to which the radial glial processes attach [30, 31]; however, the mRNA levels of Itgb1, Itga5, and Itga6 did not differ (Additional file 3: Figure S3J-L). We did not observe changes in the expression of genes in the BMP, AKT/PKB, Cdc42, GSK3 $\beta$, integrin or reelin signalling pathways in the $\mathrm{Fstl1}^{-/-}$mice, suggesting that FSTL1 may regulate radial glial development through a unique mechanism. Further study is required to elucidate this mechanism.

\section{Discussion}

In the present study, we report that Fstl1 is involved in the development of radial glial basal processes and in 


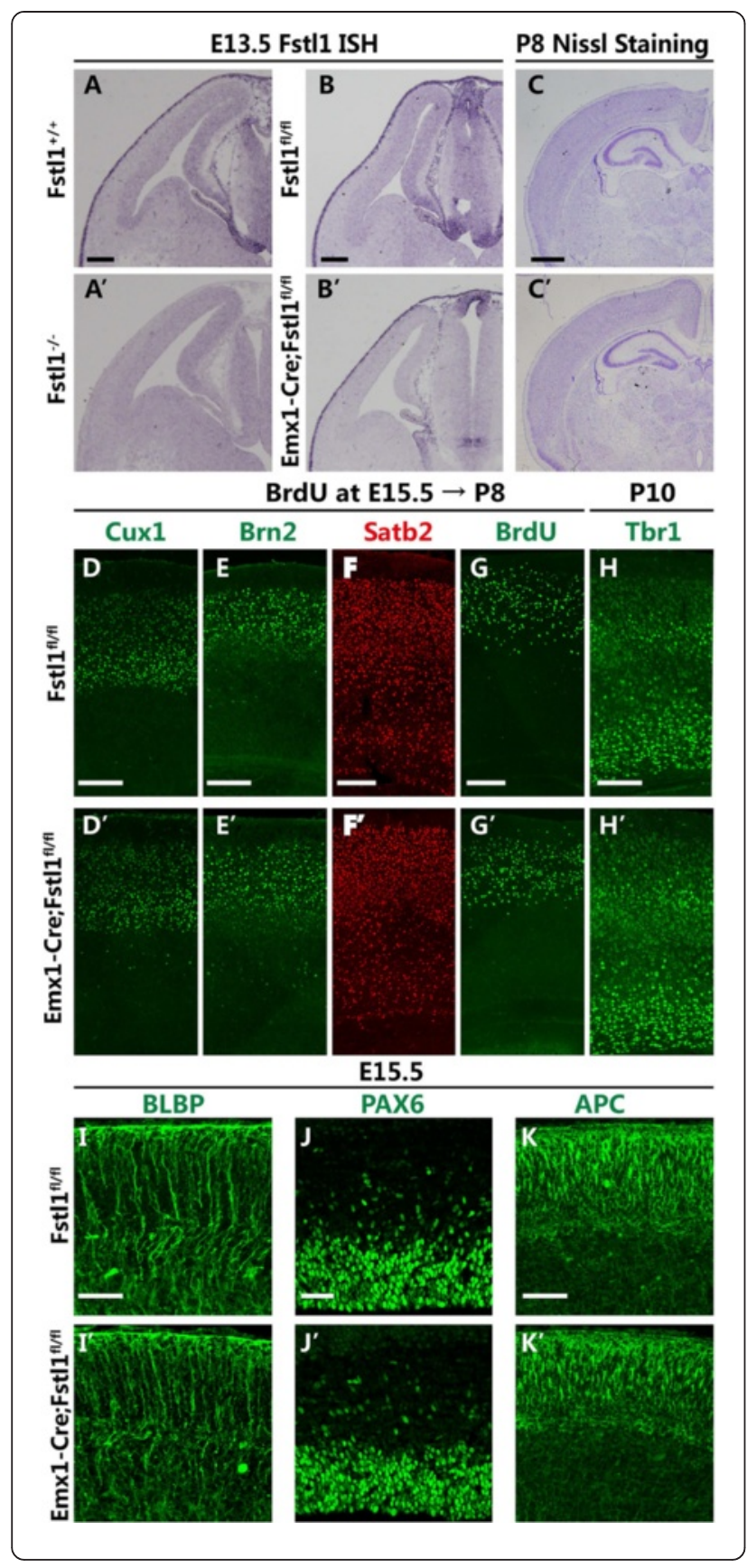

Fig. 8 Fst/1 deletion in the VZ only was not sufficient to induce radial glial dysplasia. $\mathbf{a}$ to $\mathbf{b}^{\prime}$ A high level of Fst/1 mRNA can be detected in the pia mater and VZ in the WT (a) and Fst/7 ${ }^{f / / 7}$ (b) cortices. In the Fst $/ 1^{-/-}$ mice, Fst/1 mRNA cannot be detected in the pia mater or the cerebral

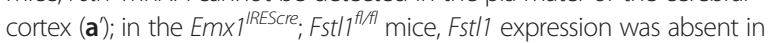
the VZ but present in the pia mater ( $\left.\mathbf{b}^{\prime}\right)$. $\mathbf{c}$ and $\mathbf{c}^{\prime}$ Nissl staining showed no remarkable differences in the structure or cellular distribution of the

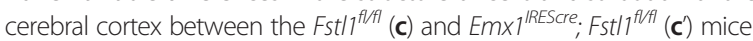
at P8. $\mathbf{d}$ to $\mathbf{h}^{\prime}$ Immunolabelling with anti-Cux1 (d and d $\mathbf{d}$ ), anti-Brn2 (e and $\mathbf{e}^{\prime}$ ) and anti-Satb2 (f and $\mathbf{f}^{\prime}$ ) antibodies at P8 and with anti-Tbr1 ( $\left(\mathbf{h}\right.$ and $\mathbf{h}^{\prime}$ ) antibodies at P10 showed that both the upper- (d to $\mathbf{f}^{\prime}$ ) and the deeper-layer ( $\mathbf{h}$ and $\mathbf{h}^{\prime}$ ) cortical neurons were nicely laminated and eventually migrated to the appropriate cortical layers in the $E m \times 1^{\text {RESCre, }}$

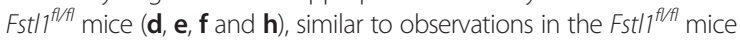
$\left(\mathbf{d}^{\prime}, \mathbf{e}^{\prime}, \mathbf{f}^{\prime}\right.$ and $\mathbf{h}^{\prime}$ ). $\mathbf{g}$ and $\mathbf{g}^{\prime}$ There were no differences in the final destinations of the cells that were labelled with BrdU at E15 between the $\mathrm{Em}_{\mathrm{m}} 1^{\mid \mathrm{RESCre}} ; \mathrm{Fst} / 7^{\mathrm{fl} / \mathrm{fl}}$ and $\mathrm{Fst} / 7^{\mathrm{fl} / \mathrm{fl}}$ cortices at P8. $\mathbf{i}$ and $\mathbf{i}^{\prime}$ BLBP immunostaining showed normal radial glial development in the

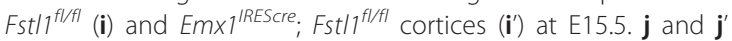
Pax6 immunostaining showed no obvious differences between the

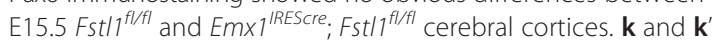
Normal basal branching of the radial glial, as demonstrated by

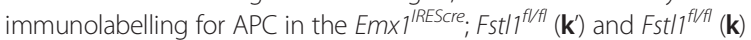
cortices. Scale bars: $100 \mu \mathrm{m}$ for $\mathbf{a}-\mathbf{b}^{\prime} ; 200 \mu \mathrm{m}$ for $\mathbf{c}$ to $\mathbf{h}^{\prime} ; 50 \mu \mathrm{m}$ for $\mathbf{i}-\mathbf{k}$

the migration of upper-layer pyramidal neurons in the developing cerebral cortex. Furthermore, the ablation of FSTL1 in both the VZ and the pia mater rather than only in the VZ led to abnormal RGC morphology, indicating that VZ-derived FSTL1 is not necessary for the development of RGC scaffolds and that FSTL1 derived from the pia mater is more important.

The role of Fst/1 in the development of radial glial scaffolds Previous studies have demonstrated that proper apicalbasal polarity and tight anchorage to the pial BM are critical for the functions of RGCs [5, 7, 8, 32]. Radial glial development has been reported to be regulated by both intrinsic factors, such as asymmetric protein distribution, and extrinsic factors, such as growth factors and other diffusible ligands $[8,33,34]$. In recent years, several studies have shown that the meninges regulate the survival, proliferation, differentiation and neurogenic properties of RGCs by releasing diffusible factors, such as CXCL12, BMP7 and retinoic acid [9, 23, 35]. Here, we found that Fstl1 is required for the development of radial glial scaffolds and the basal polarity of RGCs. Interestingly, the disruption of FSTL1 in the VZ did not affect the morphology of radial glial scaffolds, indicating that FSTL1 derived from the pia mater rather than the VZ might play a more critical role during this process and that the role of Fstl1 in the development of radial glial scaffolds is most likely non-cell-autonomous. Additionally, previous studies have suggested that meningeal cells organize the pial BM, a critical anchor point for radial glial scaffolds. Impaired pial BM integrity leads to RGC scaffold 
detachment and the apoptotic death of RGCs. However, our data indicates that Fstl1 is not indispensable for the integrity of the pial $\mathrm{BM}$ during the overall embryonic stage. One possible explanation is that FSTL1 produced by the meninges might be critical for the interaction of RGC basal endfeet with the BM, although we cannot exclude the possibility that FSTL1 regulates the organization of the RGC scaffolds directly. Further studies that employ conditional disruption of Fstl1 in the pial BM will help to elucidate the details of the role of Fstl1 in the pial BM.

The finding that RGC proliferation was not affected by the ablation of Fstl1 was also notable. Furthermore, neither the RGC progenitor pool nor the number of IPCs was disturbed, which indicates that the differentiation of RGCs was also unaffected. Our findings demonstrate a new role of Fstl1 in the maintenance of radial glial scaffolds but not the proliferation and differentiation of RGCs and thus will increase understanding of the molecular mechanisms of RGC development.

\section{Fstl1 is required for glial-guided neuronal migration}

Our results show that the deletion of Fstl1 led to mispositioning of upper-layer projection neurons. Notably, at E18.5, the distributions of deeper-layer projection neurons were not obviously affected. The lamination of layers V and VI seemed normal. One possible explanation is that neuronal migration of all layer neurons, including the upper- and deeper-layer projection neurons, has been delayed. However, the results of our birth-dating and immunostaining of deeper-layer neurons at earlier embryonic stages demonstrated that the migration of deeper-layer neurons was normal after the ablation of Fstl1, indicating that Fstl1 is not required for the migration of deeper-layer projection neurons.

Upper-layer neurons are known to preferentially migrate to their final location in a radial-glia-dependent manner, but the deeper-layer neurons likely migrate in a radialglia-independent manner [36, 37]. Thus, the impaired upper-layer projection neuron migration in the $\mathrm{Fstll}^{-1-}$ mice was probably the consequence of the abnormalities in the RGCs. It is well known that cortical interneurons migrate tangentially from the ventral telencephalon to the neocortex in a radial-glia-independent manner during embryonic development and then reach their final destination postnatally by radial migration. Here, no distribution defects were observed in our mutants, suggesting that Fstl1 is not important for the development of interneurons. Our data will help to elucidate the mechanisms of upper-layer neuronal migration.

The possible molecular mechanism of Fst/1 regulating the development of radial glial scaffolds

Recently, several studies have revealed that BMP signalling and the AKT pathway are the main downstream targets of
FSTL1 [20, 21, 26, 38, 39]. However, in the present study, no changes were detected in either pathway after the ablation of Fstl1. Reelin is a key regulator of the development of RGCs [29, 40]. However, reelin expression was also unchanged. A likely explanation is that FSTL1 does not function via either the reelinmediated pathway or the BMP or AKT pathways, and further studies are required to elucidate the molecular mechanism.

\section{Conclusion}

In the present study, we identified a new role for FSTL1 in the development of radial glial scaffolds and glialguided neuronal migration. Disruption of Fstl1 resulted in an abnormal distribution of cortical upper-layer neurons, which was due to the disruption of radial glial scaffold development. In the absence of Fstl1, the basal rather than the apical polarity of RGCs was altered. Our $\mathrm{Fstl1}^{-/-}$mice showed no changes in the expression of the genes of the BMP, AKT/PKB, Cdc42, GSK3 $\beta$, integrin or reelin signalling pathways, indicating that FSTL1 may function through a unique mechanism. Our findings will improve understanding of the molecular mechanisms of RGC development and cortical neuronal migration. Further study is required to elucidate the mechanism.

\section{Materials and methods}

\section{Animals and tissue collection}

Conventional disruption of Fstl1 was achieved by crossing Fstl ${ }^{f l /+}$ with EIIa-Cre to generate $\mathrm{Fstl}^{+/-}$mice, and the $F s t l 1^{-/-}$mice were obtained by intercrossing the $F s t l 1^{+/-}$mice $[20,41]$. Conditional disruption of Fstl1 in the VZ was achieved by crossing Fstll $P^{f l f l}$ mice with Emx $1^{\text {IREScre }}$ mice [19] (purchased from Jackson Laboratory, Bar Harbor, Maine, USA). All animals were bred in the animal facility at Southeast University. All experiments were performed according to guidelines approved by Southeast University. Embryo gender was not considered in this study. The morning of plug detection was considered to be embryonic day 0.5 (E0.5), and the day of birth was considered to be postnatal day 0 (P0).

\section{Nissl and haematoxylin-eosin staining assay}

Brain tissue was prepared as previously described [42], and sectioning was performed using a freezing microtome (Leica, CM 1950, 8-25 $\mu \mathrm{m}$ thick). Haematoxylin-eosin staining and Nissl staining were performed according to standard protocols.

\section{RNA extraction and CDNA synthesis}

For E14.5 and E18.5 embryos, the dorsal cerebral cortices were dissected out, while for E12.5 embryos, the total telencephalon was removed. The tissue was immediately 
Table 1 Primers for quantitative real-time PCR

\begin{tabular}{lll}
\hline & Forward & Reverse \\
\hline AKT1 & CGGATACCATGAACGACGTAG & GCAGGCAGCGGATGATAAAG \\
BMP7 & GAAGTCCATCTCCGTAGTATCCG & TCTGGTCACTGCTGCTGTTा \\
Cdc42 & GTTGGTGATGGTGCTGTTG & CTGTGGATAACTTAGCGGTCG \\
Etv5 & AAGAGGTTGCTCGCCGTTG & CCTTCTGCATGATGCCCTIT \\
Fstl1 & GCTCCCACCTTCGCCTCTAA & CCCTGCCAGCTCCACAAAA \\
GSK3b & TTGTCTGCCGAAATGAGTGA & GTTGCTTGGGCTTGCT \\
Itga5 & GACCTGGGCTTAGAAACCTATT & TGAGGTTCCAGGTCTGTTTG \\
Itga6 & TGGCCTTCTTTCTCCATCTC & CTGTCGACCCTGTGCTTA \\
Itgb1 & GACAGTGTGTGTGTAGGAAGAG & GCCTCCACAAATAAGCCATTAG \\
Reelin & CCCAGCCCAGACAGACAGTT & CCAGGTGATGCCATTGTTGA \\
\hline
\end{tabular}

transferred to $500 \mu \mathrm{L}$ of Trizol (Invitrogen) and processed for total RNA isolation according to the manufacturer's protocol. After the RNA was purified with the RNeasy Plus Mini Kit (QIAGEN), the concentration and integrity of the RNA were analysed with the Agilent 2100 Bioanalyser (Agilent Technologies, Palo Alto, CA). The cDNA was synthesized using the M-MLV Version cDNA Synthesis Kit (Takara, Dalian, China) from $2 \mu \mathrm{g}$ of purified total RNA.

\section{Quantitative real-time polymerase chain reaction}

qRT-PCR was performed according to standard methods as previously described [42], and total RNA from at least three different embryos of each genotype was analysed. Specific primers for Fstl1, Etv5, AKT1, Cdc42, GSK3b, BMP7, Itga5, Itga6, and Itgb1 were used for qRT-PCR and are listed in Table 1.

\section{In situ hybridization}

The in situ hybridization was performed as previously described [12], and sections from at least three different animals of each genotype were analysed. The cDNAs were obtained as previously described. The probes for Fstl1 and Etv 5 were amplified using the following primers: Fstl1: 5'-AAGGAAAAAAGCGGCCGCCCCACCTTCGC CTCTA-3' and 5'-ACGCGTCGACATAAGATTCGCTG CCATACA-3'; and Etv5: 5'-CCGGAATTCCTGTGCTG ACTCAGAAGTGCCTAAC-3' and 5'-ACGCGTCGACA GTAAGCGAAGCCTTCGGTGTAGGG-3'.

\section{Dil tracing analysis}

For each genotype, at least six embryonic brains were fixed in $4 \%$ PFA for $4-8 \mathrm{~h}$. Two to three microliters of DiI (1,1'-dioctadecyl 3,3,3',3'-tetramethylindocarbocyanine perchlorate; Invitrogen; 5 \% in DMSO) were injected into the lateral ventricle and allowed to diffuse at $37^{\circ} \mathrm{C}$ for 7 days. Vibratome sections $(150 \mu \mathrm{m})$ were obtained. The images include a projection of an $80-\mu \mathrm{m}$ z-stack collected using a $20 \times$ or $40 \times$ objective on an Olympus FV1000 confocal microscope and those acquired on a standard fluorescence microscope (Olympus BX61, Tokyo, Japan).

\section{Immunofluorescence}

Immunofluorescence experiments were performed as previously described [42]. For each genotype, at least three histological sections at three distinct rostrocaudal levels from three different animals were analysed for each immunostaining, and confocal optical sections were acquired. The primary antibodies and dilutions were as follows: rabbit anti-APC (Abcam, ab15270, 1:100), anti-BLBP (Abcam, ab32423, 1:1000), anti-CDP (M-222, Santa Cruz Biotechnology, Santa Cruz, CA, 1:500), anti-cleaved caspase-3 (Cell Signaling Technology, 9661, 1:500), anti-Ctip2 (Abcam, ab28448, 1:1000), anti-laminin A (Sigma-Aldrich, L9393, 1:2000), antiPax6 (Covance, 1:1000), anti-Tbr1 (Abcam, ab31940, 1:1000), and anti-Tbr2 (Abcam, ab23345, 1:800); mouse anti-beta-catenin (BD Biosciences, 1:500), antiCOUP-TFII (R\&D, PP-H7147-00,2ZH7147H, 1:1000), anti-Ki67 (Leica, NCL-L-Ki67-MM1, 1:100), antinestin (Developmental Studies Hybridoma Bank [DSHB], Rat-401, 1:150), anti-reelin (Millipore, MAB5364, 1:3000), anti-SATB2 (Santa Cruz Biotechnology, Santa Cruz, CA, sc-81376, 1:500), and anti-ZO-1 (Invitrogen, 339100, 1:200); rat anti-BrdU (Abcam, ab6326, 1:5000), anti-histone H3 (phospho S28) (Abcam, ab10543, 1:1000), and anti-Ctip2 (Abcam, ab18645, 1:1000); and goat antiBrn2 (C-20, Santa Cruz Biotechnology, Santa Cruz, CA, sc-6029, 1:50). The secondary antibodies included DyLight 488 donkey anti-rabbit (Thermo Fisher Scientific, SA510038, 1:500), Alexa Fluor 488 goat anti-rabbit (Invitrogen, A11008, 1:500), DyLight 488 donkey anti-goat (Thermo Fisher Scientific, SA5-10086, 1:500), DyLight 488 donkey anti-mouse (Thermo Fisher Scientific, SA5-10066, 1:500), Alexa Fluor 488 goat anti-rat (Invitrogen, A11006, 1:500), Alexa Fluor 555 donkey anti-rabbit (Invitrogen, A31572, 1:500), DyLight 550 donkey anti-mouse (Thermo Fisher Scientific, SA5-10067, 1:500), Alexa Fluor 546 goat anti-rat (Invitrogen, A11081, 1:500), and DyLight 650 donkey antirabbit (Thermo Fisher Scientific, SA5-10041, 1:500).

\section{BrdU analysis and birth-dating experiments}

The thymidine analogue BrdU (Sigma-Aldrich, St. Louis, $\mathrm{MO})$ was injected intraperitoneally into pregnant females at E12.5, E14.5 or E15.5 at a concentration of $50 \mathrm{mg} / \mathrm{kg}$ body weight. For acute BrdU labelling, embryonic brains were harvested $1 \mathrm{~h}$ later. For birth-dating, brains were harvested at E18.5 or P8. For BrdU immunolabelling, 8- to 25- $\mu \mathrm{m}$ sections were acid-treated with $2 \mathrm{M} \mathrm{HCl}$ for $25 \mathrm{~min}$ at $37{ }^{\circ} \mathrm{C}$, carefully washed in $0.1 \mathrm{M}$ PBS, and stained as described above with a monoclonal rat antibody to BrdU (Abcam, ab6326, 1:5000). 


\section{Western blotting}

The E14.5 or E12.5 dorsal cerebral cortices were collected and homogenized. The brain lysates from at least four embryos of each genotype were clarified by centrifugation at $14000 \mathrm{rpm}$. The protein concentrations were measured (Pierce Biotechnology, Rockford, IL). The brain lysates $(20 \mu \mathrm{g})$ were subjected to $10 \%$ SDS-PAGE and transferred to PVDF membranes. After the membranes were blocked with $5 \%$ non-fat dry milk in Tris-buffered saline with $0.5 \%$ Tween-20, they were incubated at $4{ }^{\circ} \mathrm{C}$ with primary antibodies, followed by incubation with the HRP-linked anti-rabbit IgG secondary antibody (Cell Signaling Technology, 7074, 1:8000) and SuperSignal West Pico Chemiluminescent Substrate (Thermo Scientific) detection. The primary antibodies were as follows: rabbit anti-phospho-Smad1 (Ser463/465)/Smad5 (Ser463/465)/ Smad8 (Ser426/428) (Cell Signaling Technology, 9511, 1:4000); rabbit anti-phospho-AKT (Ser473) (Cell Signaling Technology, 4060, 1:4000); rabbit anti-AKT (Cell Signaling Technology, 9272, 1:5000); and rabbit anti-GAPDH (Cell Signaling Technology, 2118, 1:5000).

\section{Microscopic analysis}

The images were acquired on an Olympus (Tokyo, Japan) BX61 microscope equipped with appropriate filter sets and a digital camera (DP71) or on an Olympus FV1000 confocal microscope and were processed using Image-Pro Plus 6.0.

\section{Morphometric analysis and statistics}

For morphometric analysis, at least three embryos were analysed for each condition in parallel experiments. Both hemispheres of at least three matching sections from each brain were used for the measurements, and comparisons between the mutants and the control littermates were made using more than three litters. The cells were counted in a radial segment that was 200 or $300 \mu \mathrm{m}$ in width and spanned from the ventricular surface to the pia. For the proliferation assay, the number of $\mathrm{BrdU}^{+}$cells in each radial unit was counted. For the birth-dating experiments, a single optical section of a confocal microscope image was used, and the number of $\mathrm{BrdU}^{+}$cells in ten equally divided bins that spanned the cortical thickness was counted and expressed as a fraction of the total number of $\mathrm{BrdU}^{+}$cells per bin. To evaluate the distribution of $\mathrm{Cux}^{+}{ }^{+}$neurons, sections were divided into ten bins of equal height and $300 \mu \mathrm{m}$ in width. Bins 1-3, 4-6 and 7-9 were grouped as upper, middle and lower, respectively. All of the data were statistically analysed with Microsoft Office Excel and graphed using GraphPad Prism software. The error bars represent the standard error of the mean. Two-tailed Student's $t$-test was used to analyse statistical significance (" $P \leq 0.05$, ** $P \leq 0.01$, *** $P \leq 0.001)$.

\section{Additional files}

Additional file 1: Figure S1. The neurogenesis of upper-layer neuron was not affected by Fst/1 deletion. (A to D') IPCs at E13.5 (A to B'), E14.5 ( $C$ to $C^{\prime}$ ) and E15.5 (D to $D^{\prime}$ ) were labelled by immunostaining with an anti-Tbr2 antibody. The number and distribution of the $\mathrm{Tbr} 2^{+} \mathrm{IPCs}$ were similar between the Fst/1 ${ }^{-1-}\left(A^{\prime}, B^{\prime}, C^{\prime}\right.$ and $\left.D^{\prime}\right)$ and the WT $(A, B, C$ and $D)$ cortices. Scale bars: $100 \mu \mathrm{m}$. (PDF $420 \mathrm{~kb}$ )

Additional file 2: Figure S2. The integrity of the pial BM was not affected by the deletion of Fst/1. (A to $A^{\prime}$ ) A robust, continuous band of laminin A immunoreactivity was detected in both the $\mathrm{Fst}_{\mathrm{I}} \mathrm{1}^{-1-}\left(\mathrm{A}^{\prime}\right)$ and WT (A) cortices. Scale bars: $50 \mu \mathrm{m}$. (PDF $53 \mathrm{~kb}$ )

Additional file 3: Figure S3. Analysis of the possible mechanisms by which Fst/1 regulates the development of radial glial processes. (A) Western blotting for phospho-Smad1/5/8, AKT and phospho-AKT (S473). Similar phosphorylation levels of Smad1/5/8 and AKT in the Fst/1 ${ }^{-/}$and WT brains at E14.5. The expression of AKT in the Fst/1 ${ }^{-1-}$ cortices did not differ from that in the WT cortices. (B to C) Quantification of the mRNA levels of BMP7 (B) and AKT1 (C) at E14.5 showed no differences. (D and D') Etv5 mRNA was predominantly expressed at E14.5 in the $\mathrm{VZ}$ of the $\mathrm{Fst}_{\mathrm{t}} 1^{-1-}$ mice (D'), similar to the expression in the WT mice (D). (E and F) The transcription levels of Etv5 (D) and reelin (F) mRNA did not differ between the WT and $\mathrm{Fst}_{1} 1^{-1-}$ mice. (F) ( $G$ and $\mathrm{G}^{\prime}$ ) The distribution of reelin ${ }^{+} \mathrm{CR}$ cells in the $\mathrm{Fst}_{1} 1^{-1-}$ mice also did not differ from that in the WT mice. ( $H$ to $L)$ Quantification of the mRNA levels of Cdc42 (H), GSK3 $\beta$ (I) and integrinß1/a5/a6 (J to L) by RT-PCR showed similar mRNA levels in the WT and Fst $11^{-1-}$ mice. Scale bars: $50 \mu \mathrm{m}$ for D and D'; $100 \mu \mathrm{m}$ for $\mathrm{F}$ and $\mathrm{F}^{\prime}$. (PDF $723 \mathrm{~kb}$ )

\section{Abbreviations}

APC: Adenomatosis polyposis coli; BLBP: Brain lipid-binding protein; BM: Basement membrane; BMP: Bone morphogenetic protein; BrdU: 5bromo-2-deoxyuridine; Brn2: POU domain transcription factor 2; cdc42: Cell division cycle 42; CGE: Caudal ganglionic eminence; COUP-TFIl: Ovalbumin upstream promoter transcription factor II; CR: Cajal-Retzius; Ctip2: Chicken ovalbumin upstream promoter transcription factor-interacting protein 2; Cux1: Cut-like homeobox 1; CXCL12: Chemokine (C-X-C motif) ligand 12; Etv5: Ets variant 5; FGF: Fibroblast growth factor; Foxp2: Forkhead box protein 2; Fstl1: Follistatin like-1; GAPDH: Glyceraldehyde-3-phosphate dehydrogenase; GSK3 3 : Glycogen synthase kinase 3 beta; HRP: Horseradish peroxidase; IPCs: Intermediate progenitor cells; Itga: Integrin alpha; Itgb1: Integrin beta-1; IZ: Intermediate zone; Lhx6: LIM homeobox protein 6; MAPK: Mitogen-activated protein kinase; MGE: Medial ganglionic eminence; PAGE: Polyacrylamide gel electrophoresis; Pax6: Paired box gene 6; pHH3: Phospho-histone H3; PKB: Protein kinase B; PVDF: Polyvinylidene difluoride; RGCs: Radial glial cells; SDS: Sodium dodecyl sulphate;

SST: Somatostatin; Tbr1: T-box brain gene 1; Tbr2: T-box brain gene 2; VZ: Ventricular zone; ZO-1: Tight junction protein 1.

\section{Competing interests}

The authors declare that they have no competing financial interests.

\section{Authors' contributions}

$C Z$ conceived the study; $C Z$ and $R L$ designed the research; $R L$ and $Y Y$ performed the immunostaining and in situ hybridization and analysed the data; JHS, QQZ, RB, and YJW performed the statistical analyses; HC and RL performed the Nissl staining; RL performed the western blotting, quantitative real-time PCR and Dil-tracing analyses; KCL and XZ generated the Fst $/ 7^{\text {fl/fl }}$ mouse line; and RL and CZ wrote the paper. All authors read and approved the final manuscript.

\section{Acknowledgements}

We thank Dr Xiang Gao (Model Animal Research Centre, Nanjing University) for providing the Fstf fl/fl and Ella-Cre mouse lines, Yiquan Wei and Li Liu for their assistance with laboratory and animal care and other members of the laboratory for discussion. 


\section{Funding}

This work was supported by the National High Technology Research and Development Program (2012AA022402) and the National Natural Science Foundation of China (91232301 and 31171040 to C. Z.).

\section{Author details}

${ }^{1}$ Key Laboratory of Developmental Genes and Human Diseases, MOE, Department of Anatomy and Neuroscience, Medical School, Southeast University, Nanjing 210009, China. ${ }^{2}$ Institute of Neuroscience and State Key Laboratory of Neuroscience, Shanghai Institutes for Biological Sciences, Chinese Academy of Sciences, Shanghai 200031, China. ${ }^{3}$ Center of Depression, Beijing Institute for Brain Disorders, Beijing 100069, China.

\section{Received: 27 April 2015 Accepted: 28 August 2015}

\section{Published online: 17 September 2015}

\section{References}

1. Rakic P. Developmental and evolutionary adaptations of cortical radial glia. Cereb Cortex. 2003;13:541-9.

2. Anthony TE, Klein C, Fishell G, Heintz N. Radial glia serve as neuronal progenitors in all regions of the central nervous system. Neuron. 2004;41:881-90

3. Barry DS, Pakan JM, McDermott KW. Radial glial cells: key organisers in CNS development. Int J Biochem Cell Biol. 2014;46:76-9.

4. Wrobel CN, Mutch CA, Swaminathan S, Taketo MM, Chenn A. Persistent expression of stabilized beta-catenin delays maturation of radial glial cells into intermediate progenitors. Dev Biol. 2007;309:285-97.

5. Yokota Y, Eom TY, Stanco A, Kim WY, Rao S, Snider WD, et al. Cdc42 and Gsk3 modulate the dynamics of radial glial growth, inter-radial glial interactions and polarity in the developing cerebral cortex. Development. 2010;137:4101-10.

6. Wang X, Imura T, Sofroniew MV, Fushiki S. Loss of adenomatous polyposis coli in Bergmann glia disrupts their unique architecture and leads to cell nonautonomous neurodegeneration of cerebellar Purkinje neurons. Glia. 2011;59:857-68

7. Yokota Y, Kim WY, Chen Y, Wang X, Stanco A, Komuro Y, et al. The adenomatous polyposis coli protein is an essential regulator of radial glial polarity and construction of the cerebral cortex. Neuron. 2009;61:42-56.

8. Radakovits R, Barros CS, Belvindrah R, Patton B, Muller U. Regulation of radial glial survival by signals from the meninges. J Neurosci. 2009;29:7694-705.

9. Siegenthaler JA, Ashique AM, Zarbalis K, Patterson KP, Hecht JH, Kane MA, et al. Retinoic acid from the meninges regulates cortical neuron generation. Cell. 2009;139:597-609.

10. Sylva M, Moorman AF, van den Hoff MJ. Follistatin-like 1 in vertebrate development. Birth Defects Res C Embryo Today. 2013;99:61-9.

11. Umezu T, Yamanouchi H, lida Y, Miura M, Tomooka Y. Follistatin-like-1, a diffusible mesenchymal factor determines the fate of epithelium. Proc Natl Acad Sci U S A. 2010;107:4601-6.

12. Yang Y, Liu J, Mao H, Hu YA, Yan Y, Zhao C. The expression pattern of Follistatin-like 1 in mouse central nervous system development. Gene Expr Patterns. 2009;9:532-40.

13. Englund C, Fink A, Lau C, Pham D, Daza RA, Bulfone A, et al. Pax6, Tbr2, and Tbr1 are expressed sequentially by radial glia, intermediate progenitor cells, and postmitotic neurons in developing neocortex. J Neurosci. 2005;25:247-51.

14. Kriegstein AR, Noctor SC. Patterns of neuronal migration in the embryonic cortex. Trends Neurosci. 2004;27:392-9.

15. Heins N, Malatesta P, Cecconi F, Nakafuku M, Tucker KL, Hack MA, et al. Glial cells generate neurons: the role of the transcription factor Pax6. Nat Neurosci. 2002;5:308-15.

16. Siegenthaler JA, Pleasure SJ. We have got you 'covered': how the meninges control brain development. Curr Opin Genet Dev. 2011;21:249-55.

17. Chae TH, Kim S, Marz KE, Hanson PI, Walsh CA. The hyh mutation uncovers roles for alpha Snap in apical protein localization and control of neural cell fate. Nat Genet. 2004;36:264-70.

18. Bultje RS, Castaneda-Castellanos DR, Jan LY, Jan YN, Kriegstein AR, Shi SH. Mammalian Par3 regulates progenitor cell asymmetric division via notch signaling in the developing neocortex. Neuron. 2009;63:189-202.

19. Gorski JA, Talley T, Qiu M, Puelles $L$, Rubenstein JL, Jones KR. Cortical excitatory neurons and glia, but not GABAergic neurons, are produced in the Emx1-expressing lineage. J Neurosci. 2002;22:6309-14.
20. Geng Y, Dong Y, Yu M, Zhang L, Yan X, Sun J, et al. Follistatin-like 1 (Fstl1) is a bone morphogenetic protein (BMP) 4 signaling antagonist in controlling mouse lung development. Proc Natl Acad Sci U S A. 2011;108:7058-63.

21. Xu J, Qi X, Gong J, Yu M, Zhang F, Sha H, et al. Fstl1 antagonizes BMP signaling and regulates ureter development. PLoS One. 2012;7:e32554.

22. Ortega JA, Alcantara S. BDNF/MAPK/ERK-induced BMP7 expression in the developing cerebral cortex induces premature radial glia differentiation and impairs neuronal migration. Cereb Cortex. 2010;20:2132-44.

23. Segklia A, Seuntjens E, Elkouris M, Tsalavos S, Stappers E, Mitsiadis TA, et al. Bmp7 regulates the survival, proliferation, and neurogenic properties of neural progenitor cells during corticogenesis in the mouse. PLoS One. 2012; 7:e34088.

24. Liu S, Shen H, Xu M, Liu O, Zhao L, Liu S, et al. FRP inhibits ox-LDL-induced endothelial cell apoptosis through an Akt-NF-\{kappa\}B-Bcl-2 pathway and inhibits endothelial cell apoptosis in an apoE-knockout mouse model. Am J Physiol Endocrinol Metab. 2010;299:E351-63.

25. Liu S, Wang L, Wang W, Lin J, Han J, Sun H, et al. TSC-36/FRP inhibits vascular smooth muscle cell proliferation and migration. Exp Mol Pathol. 2006;80:132-40.

26. Oshima Y, Ouchi N, Sato K, Izumiya Y, Pimentel DR, Walsh K. Follistatin-like 1 is an Akt-regulated cardioprotective factor that is secreted by the heart. Circulation. 2008:117:3099-108.

27. Li X, Newbern JM, Wu Y, Morgan-Smith M, Zhong J, Charron J, et al. MEK is a key regulator of gliogenesis in the developing brain. Neuron. 2012;75:1035-50.

28. Soriano E, Alvarado-Mallart RM, Dumesnil N, Del Rio JA, Sotelo C. CajalRetzius cells regulate the radial glia phenotype in the adult and developing cerebellum and alter granule cell migration. Neuron. 1997;18:563-77.

29. Hartfuss E, Forster E, Bock HH, Hack MA, Leprince P, Luque JM, et al. Reelin signaling directly affects radial glia morphology and biochemical maturation. Development. 2003;130:4597-609.

30. Belvindrah R, Graus-Porta D, Goebbels S, Nave KA, Muller U. Beta1 integrins in radial glia but not in migrating neurons are essential for the formation of cell layers in the cerebral cortex. J Neurosci. 2007;27:13854-65.

31. Marchetti G, Escuin S, van der Flier A, De Arcangelis A, Hynes RO, GeorgesLabouesse E. Integrin alpha5beta1 is necessary for regulation of radial migration of cortical neurons during mouse brain development. Eur J Neurosci. 2010;31:399-409.

32. Weimer JM, Yokota Y, Stanco A, Stumpo DJ, Blackshear PJ, Anton ES. MARCKS modulates radial progenitor placement, proliferation and organization in the developing cerebral cortex. Development. 2009;136:2965-75.

33. Campbell K. Signaling to and from radial glia. Glia. 2003;43:44-6.

34. Gotz M, Stoykova A, Gruss P. Pax6 controls radial glia differentiation in the cerebral cortex. Neuron. 1998:21:1031-44.

35. Mithal DS, Ren D, Miller RJ. CXCR4 signaling regulates radial glial morphology and cell fate during embryonic spinal cord development. Glia. 2013;61:1288-305.

36. Gal JS, Morozov YM, Ayoub AE, Chatterjee M, Rakic P, Haydar TF. Molecular and morphological heterogeneity of neural precursors in the mouse neocortical proliferative zones. J Neurosci. 2006;26:1045-56.

37. Nadarajah B, Parnavelas JG. Modes of neuronal migration in the developing cerebral cortex. Nat Rev Neurosci. 2002;3:423-32.

38. Sylva M, Li VS, Buffing AA, van Es JH, van den Born M, van der Velden $\mathrm{S}$, et al. The BMP antagonist follistatin-like 1 is required for skeletal and lung organogenesis. PLoS One. 2011;6:e22616.

39. Tanaka M, Murakami K, Ozaki S, Imura Y, Tong XP, Watanabe T, et al. DIP2 disco-interacting protein 2 homolog A (Drosophila) is a candidate receptor for follistatin-related protein/follistatin-like 1-analysis of their binding with TGF-beta superfamily proteins. FEBS J. 2010;277:4278-89.

40. Lee $H$, Song MR. The structural role of radial glial endfeet in confining spinal motor neuron somata is controlled by the Reelin and Notch pathways. Exp Neurol. 2013;249:83-94.

41. Li KC, Zhang FX, Li CL, Wang F, Yu MY, Zhong YQ, et al. Follistatin-like 1 suppresses sensory afferent transmission by activating $\mathrm{Na}^{+}, \mathrm{K}^{+}-$ATPase. Neuron. 2011;69:974-87.

42. Tian C, Gong Y, Yang Y, Shen W, Wang K, Liu J, et al. Foxg1 has an essential role in postnatal development of the dentate gyrus. J Neurosci. 2012;32:2931-49. 